\title{
Visualizando Gammagramas Óseos en Colores
}

\section{Displaying Bone Scans in Color}

\author{
M. Pérez-Meza' , A. Jaramillo-Núñez² B. E. Sánchez-Rinza ${ }^{3}$ \\ 'Licenciatura en Informática, Universidad de la Sierra Sur \\ 2Instituto Nacional de Astrofísica, Óptica y Electrónica \\ ${ }^{3}$ Facultad de Ciencias de la Computación, Benemérita Universidad Autónoma de Puebla
}

\section{RESUMEN}

En este trabajo se describe el uso de un software para detectar metástasis óseas en gammagramas cuyo funcionamiento está basado en un método cuantitativo. En la investigación se incluyeron cuarenta y tres gammagramas óseos de pacientes con cáncer de próstata, los cuales fueron previamente analizados visualmente por tres especialistas y su diagnóstico se tomó como referencia. Debido a que no todos los huesos de un paciente muestran los mismos tonos de gris, cada uno de los gammagramas se segmentó para su análisis en cuatro regiones: cráneo, hombros, tórax y pelvis. La segmentación se hizo con el fin de obtener intervalos de tonos de gris en cada una de las regiones empleando un proceso estadístico. Para ello se calcularon la media y la desviación estándar de cuatro muestras que contenían cada una cuarenta y tres regiones. Tomando en consideración los intervalos, siete colores fueron asignados a cada región. Mediante los colores fue posible diferenciar los casos sanos e infiltrados en cada región, lo que facilita el diagnóstico. Se muestran ejemplos de los resultados en cada una de las regiones.

PALABRAS ClAVE: gammagrama óseo; metástasis óseas; cáncer de próstata; procesado digital de imágenes 


\section{ABSTRACT}

The use of software based on a quantitative method for detecting skeletal metastasis in bone scans is presented. Forty-three bone scans of patients with prostate cancer were previously analyzed visually by three specialists and their diagnosis were taken as a reference. Later, these scans were analyzed by segmenting them into four regions: skull, shoulders, chest, and pelvis. The segmentation was made to obtain intervals of gray levels for each of the regions using a statistical process. These values were found by calculating the mean and standard deviation of four samples containing each forty-three regions. Using these intervals, seven colors were assigned to each region. By means of the colors it is possible to differentiate healthy and infiltrated cases in each region, which may facilitate the diagnosis. Examples of results in each region are shown.

KEYWORDS: bone scan; prostate cancer; skeletal metastasis; digital image processing

\section{Correspondencia}

DESTINATARIO: Mónica Pérez Meza

INSTITUCIÓN: Licenciatura en Informática,

Universidad de la Sierra Sur

DIRECCIÓN: Guillermo rojas Mijangos S/N, esquina

Avenida Universidad, Col. Ciudad Universitaria, C. P. 70800, Miahuatlán de Porfirio Díaz, Oaxaca, México CORREO ELECTRÓNICO: arielmonp@hotmail.com

\section{Fecha de recepción:}

16 de marzo de 2018

Fecha de aceptación:

13 de julio de 2018 


\section{INTRODUCCIÓN}

La gammagrafía ósea es la modalidad de imagen más común empleada en medicina nuclear para evaluar la dispersión metastásica del cáncer a hueso. Su interpretación se realiza visualmente y para dar diagnósticos acertados se requiere experiencia debido a las dificultades asociadas con el reconocimiento de los puntos calientes. Por lo tanto, una interpretación cuantitativa más que cualitativa ayudaría más en la comprensión del gammagrama con el fin de mejorar y estandarizar los diagnósticos ${ }^{[1]}$.

Dos de los programas de computación más reportados en la literatura para cuantificar lesiones metastásicas son BONENAVI y EXINI. El primero usa el índice de escaneo óseo el cual consiste en analizar el gammagrama óseo para estimar las áreas enfermas de metástasis en cada hueso para posteriormente sumar todas las áreas halladas. Después, el resultado se compara con el área total de los huesos sanos y por último se realiza un cociente para determinar el porcentaje de involucramiento esquelético total ${ }^{[2]}$.

El segundo, está basado en el uso de técnicas de procesado digital de imágenes y de redes neuronales artificiales para interpretar los gammagramas óseos realizados a los pacientes con el fin de determinar la presencia o ausencia de metástasis ${ }^{[3]}$. Actualmente, ambos sistemas están completamente automatizados y son altamente eficientes, alcanzando una especificidad y sensibilidad del orden del $90 \%$.

En el mismo sentido de mejorar y estandarizar los diagnósticos se está desarrollando un software cuyo objetivo principal es determinar en gammagramas, la metástasis ósea temprana y otras enfermedades óseas tales como enfermedades degenerativas, fracturas, infecciones, inflamaciones, etc. ${ }^{[4,5]}$. Sin embargo, el trabajo que se reporta aquí es únicamente el método en el que está basado el software para determinar la presencia o ausencia de metástasis óseas.
Con ayuda del software se analizan los tonos de gris del gammagrama en regiones con alto riesgo de desarrollar metástasis óseas como son, por ejemplo, el cráneo, los hombros, el tórax y la pelvis. Los tonos de gris que puedan indicar alguna anormalidad ósea son obtenidos usando los métodos de apagado y comparación de pixeles ${ }^{[6]}$. El primer método consiste en que dado un valor inicial por el usuario, los pixeles en la imagen con valores iguales, o menores a él son sustituidos por ceros. Después, los pixeles con tonos de gris mayores que el umbral son analizados para determinar cuáles podrían indicar alguna anormalidad ósea. El segundo método consiste en comparar los tonos de gris en regiones con simetría axial, es decir, los del lado derecho con los del lado izquierdo. Por ejemplo, se comparan los tonos de gris de las vértebras del lado derecho con las del izquierdo, o los del hombro derecho con los del izquierdo, etc.

Para determinar la metástasis ósea el software usa los tonos de gris mínimo y máximo de la región de interés (ROI) de cada gammagrama. Su determinación está basada en la suposición de que un hueso sin actividad osteoblástica no absorberá radiotrazador y por lo tanto su tono de gris será cero. Sin embargo, como los huesos siempre están en constante regeneración, entonces siempre mostrarán actividad osteoblástica. Como consecuencia, sí habrá absorción de radiotrazador por lo que el tono de gris no será cero. Este dependerá de la edad del paciente, raza, género, tipo de radiofármaco usado, velocidad de escaneo, etc. En el caso de un solo paciente, la absorción de trazador y por consiguiente los tonos de gris mínimo y máximo de la ROI serán únicos, pero para una muestra dada de pacientes será un intervalo. Debido a que no todos los huesos de un paciente muestran los mismos tonos de gris, cada uno de los gammagramas se segmentó para su análisis en cuatro regiones: cráneo, hombros, tórax y pelvis. La segmentación se hizo con el fin de obtener intervalos de tonos de gris en cada una de las regiones. Tomando en consideración los intervalos, siete colores fueron 
asignados a cada región y mediante ellos diferenciamos los casos sanos de los infiltrados en cada región, lo que facilita el diagnóstico.

\section{MATERIALES Y MÉTODOS}

En el departamento de Medicina Nuclear del Centro Médico Nacional "La Raza” se realizó un estudio observacional, retrospectivo y analítico, durante el periodo comprendido del 1 de enero al 31 de diciembre de $2015^{\text {[7] }}$. El estudio se realizó empleando una muestra de 138 pacientes con cáncer de próstata con edades entre 47 y 85 años (media 68.5). La enfermedad de los pacientes fue determinada por la clasificación de Gleason y por gammagrafía ósea. Con esta última se detectó la presencia y/o ausencia de metástasis óseas mediante análisis visual.

Los gammagramas óseos se realizaron después de administrar a los pacientes una dosis de $25 \mathrm{mCi}$ de 99mTc-MDP vía intravenosa y tomar 2 litros de agua previos al registro de las imágenes. Dos horas después de la administración del radiofármaco, se les realizó un rastreo de cuerpo completo con un equipo MEDISO INTERVIEW XP VERSION 1.05014 de dos cabezales con colimador $L E H R$ a una velocidad de $12 \mathrm{~cm} / \mathrm{min}$, en proyección anterior y posterior. Posteriormente los gammagramas óseos fueron analizados visual e independientemente por tres especialistas del departamento de Medicina Nuclear y sus diagnósticos fueron usados como referencia durante la investigación.

De los 138 gammagramas óseos analizados, 43 fueron del tipo Whole-body. Debido a que fue el tipo con mayor número de gammagramas óseos en la muestra, fueron seleccionados para realizar esta investigación.

Para el análisis de los gammagramas óseos y la visualización de las regiones, la información de los archivos en formato DICOM se trabajó sin modificarse, por lo que fueron analizados directamente los "datos crudos" del archivo de la imagen ("raw" DICOM images).

\section{Desarrollo}

Con ayuda del software, cada gammagrama óseo fue segmentado en cuatro ROIs: cráneo, hombros, tórax y pelvis. Después, se hallaron los tonos de gris mínimo y máximo de la ROI de cada paciente. Estos tonos serán mencionados como el mínimo ideal (mi) y máximo ideal (MI) de la ROI. Posteriormente se calcula el promedio y la desviación estándar de los valores mi y MI de la muestra y con su ayuda se obtienen siete interva-

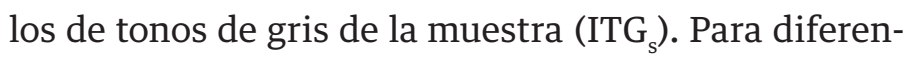
ciar los mi y MI de la ROI de los de la muestra, los últimos serán mencionados como los valores mínimo ideal $\left(\mathrm{mi}_{\mathrm{s}}\right)$ y máximo ideal $\left(\mathrm{MI}_{\mathrm{s}}\right)$ de la muestra, respectivamente. Lo anterior se realizó para las cuatro regiones en que se dividió el gammagrama.

La zona del cráneo con mayor riesgo de desarrollar metástasis es la superior, así que fue la única analizada. Los cráneos fueron clasificados en dos grupos: sanos e infiltrados, tal como lo determinaron los especialistas. Sin embargo, debido a lo observado en las imágenes los sanos fueron clasificados a su vez cómo: ideales y no ideales. Los primeros se caracterizaron principalmente por estar libres de cualquier enfermedad ósea mientras que los no ideales se caracterizaron por mostrar enfermedades óseas en progreso.

El procedimiento para hallar los valores mi y MI en la parte superior del cráneo fue el siguiente: iniciando con un valor umbral $\mathrm{u}$, dado heurísticamente, se verifica el cumplimiento de la Ec. 1

$$
\text { Si } \mathrm{rc}(\mathrm{i}, \mathrm{j})<=\mathrm{u} \text { entonces } \mathrm{rc}(\mathrm{ij})=0 \text {, en otro caso } \mathrm{u}=\mathrm{u}+1
$$

Donde rc(i,j) es la posición dentro de la imagen del pixel analizado.

Si la Ec. 1 no se cumple entonces el valor umbral se incrementa una unidad hasta hallar un valor que la satisfaga. En general, los tonos de gris de los pixeles del fondo de la imagen oscilan entre 0 y 10 por lo que un 
buen valor inicial para u podría ser 10. Si hay pixeles con valores iguales a, o menores a $\mathrm{u}$, son sustituidos por ceros. Si no hay, el valor umbral se incrementa una unidad hasta encontrar uno o más pixeles de la ROI con un valor igual al umbral. Cuando esto sucede, el valor del umbral es asignado a mi. Después, el umbral se va incrementando hasta que su valor coincide con el máximo tono de gris de cierta región particular del cráneo (esto se mostrará más adelante). Cuando esto sucede el valor del umbral es asignado a MI. El valor requerido del umbral para sustituir por ceros los pixeles restantes se le nombró apagado total (ATO), por lo cual el valor del umbral se asignó a la variable ATO. Por último, usando los valores mi, MI y ATO de cada cráneo se calculó el valor medio y la desviación estándar de la muestra. Después, empleando estos valores se hallan los ITGs de los cráneos de la muestra.

TABLA 1. Valores obtenidos de mi, MI y ATO para los cráneos ideales

\begin{tabular}{|c|c|c|c|}
\hline $\begin{array}{c}\text { No. de } \\
\text { muestra }\end{array}$ & mi & MI & ATO \\
\hline 1 & 9 & 30 & 34 \\
\hline 2 & 13 & 41 & 44 \\
\hline 3 & 15 & 56 & 60 \\
\hline 4 & 17 & 32 & 39 \\
\hline 5 & 15 & 47 & 56 \\
\hline 6 & 12 & 43 & 43 \\
\hline 7 & 10 & 38 & 38 \\
\hline 8 & 15 & 51 & 54 \\
\hline 9 & 13 & 38 & 41 \\
\hline 10 & 16 & 48 & 55 \\
\hline Media $(\overline{\mathrm{u}})$ & $\mathrm{mi}_{\mathrm{s}}=13.5$ & $\mathrm{MI}_{\mathrm{s}}=42.4$ & $\mathrm{ATO}_{\mathrm{s}}=46.4$ \\
\hline $\begin{array}{c}\text { Desviación } \\
\text { Estándar }(\sigma)\end{array}$ & 2.21 & 8.99 & 11.01 \\
\hline
\end{tabular}

La Tabla 1 muestra los valores obtenidos de mi, MI, ATO, valor medio y desviación estándar de la muestra de cráneos ideales.

El cálculo de los ITGs de las cuatro ROIs está basado en la forma en cómo se expresa el resultado de una serie de mediciones con su error, es decir, $\overline{\mathrm{u}} \pm \sigma$.
Para el cálculo de los ITGs de la Tabla 2 se usaron los valores de la media y la desviación estándar tal y como están en la Tabla 1, es decir, se consideraron los decimales. Por ejemplo, en la columna 1, renglón 2, el intervalo se halló de la siguiente manera: el valor cero es considerado como el límite inferior del ITG 1 porque es el primer tono de gris que puede contener la imagen. El límite superior del ITG 1 es calculado así: el valor de la media de la muestra (13.5) menos la desviación estándar (2.2) menos 1 da como resultado 10.3. Al redondearlo obtenemos 10 que es el límite superior del ITG 1 , es decir, el primer intervalo va de 0-10. Lo anterior es identificado en corchetes, en la columna 1, renglón 2.

TABLA 2. ITGs para la muestra de cráneos ideales

\begin{tabular}{|c|c|c|c|}
\hline No. de ITG & mi & MI & ATO \\
\hline ITG 1=[0 - (13.5-2.2-1)] & $0-10$ & & \\
\hline ITG 2 $=[11-(13.5+2.2)]$ & $11-16$ & & \\
\hline ITG 3 & & $17-32$ & \\
\hline ITG 4 & & $33-51$ & \\
\hline ITG 5 & & & $34-52$ \\
\hline ITG 6 & & & $35-57$ \\
\hline ITG 4' & & & $33-57$ \\
\hline ITG 5' & $58-124$ & & \\
\hline ITG 6' & $125-191$ & & \\
\hline ITG 7 & $192-255$ & & \\
\hline
\end{tabular}

El límite inferior del ITG 2 es 11 ya que es el entero que sigue al 10. El límite superior del ITG 2 se calcula de la siguiente manera: el valor medio de la muestra (13.5) más la desviación estándar (2.2) da como resultado 15.7, redondeando este valor obtenemos 16. De manera similar fueron calculados los intervalos 3-6. Debido a que los intervalos 4, 5 y 6 se traslapan optamos por reasignarlos a un solo intervalo, el 4'. La prima de los intervalos 4', 5' y 6' es solo para diferenciarlos de los intervalos 4, 5 y 6 . El otro motivo por el cual se escribieron así fue para mantener la numeración continua.

En la Tabla 2, el ITG más importante es el 4’ ya que este determina si los cráneos analizados pertenecen o no a esta clasificación. Los límites de los ITGs 5', 6’ y 7’ 
no fueron obtenidos en función del valor medio y la desviación estándar de la muestra sino que fueron propuestos. Para este caso particular el intervalo 58-255 (rango de tonos de gris no observado en los cráneos ideales) se dividió entre tres y el resultado se asignó a cada uno de los tres intervalos. La principal importancia de estos intervalos es mostrar rápidamente si el cráneo analizado padece de alguna anormalidad ósea, ya que si la ROI que se está analizando contiene uno o los tres colores correspondientes a los intervalos 5', 6' y 7’ automaticamente se deberá entender cómo que el cráneo observado padece de alguna anormalidad ósea.

La Figura 1 muestra cuatro imágenes obtenidas de la misma ROI, donde se observa la eliminación simétrica de los pixeles en los cráneos clasificados como ideales. La Figura 1.a muestra la sustitución por ceros de los primeros pixeles con un valor umbral de 13, el cual fue asignado a mi. La Figura 1.b fue obtenida con un umbral de 30, la Figura 1.c cuando el valor fue 36 y la Figura 1.d cuando el valor fue 38. Cuando el valor umbral fue 40 todos los pixeles de la parte superior del cráneo se sustituyeron por cero y este valor se asignó a MI. La diferencia en tonos de gris entre mi y MI fue de 27.

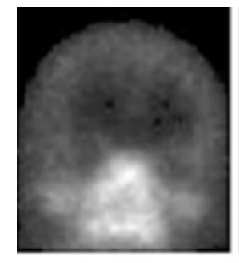

(a) 13

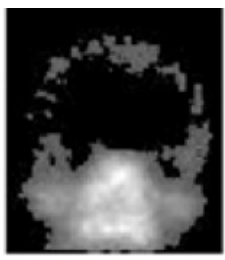

(b) 30

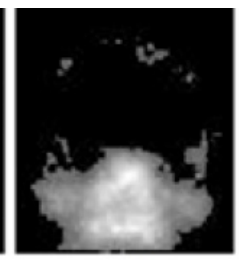

(c) 36

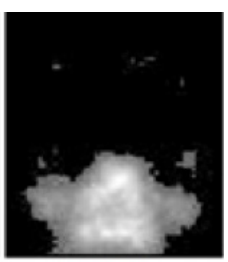

(d) 38
FIGURA 1. Eliminación homogénea

de pixeles en un cráneo ideal.

La Figura 2 muestra el comportamiento de la eliminación de los tonos de gris en un cráneo no ideal. La Figura 2.a muestra la imagen del cráneo. Los primeros dos pixeles se sustituyeron por cero cuando el valor umbral fue 21, el cual se asignó a mi. La Figura 2.b muestra el caso en el cual el umbral fue 65, valor que se asignó a MI.

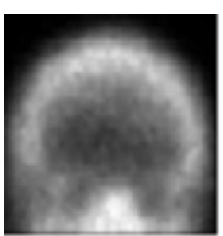

(a)

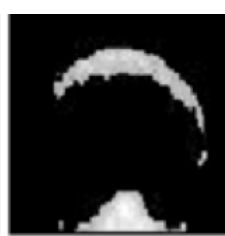

(b) 65

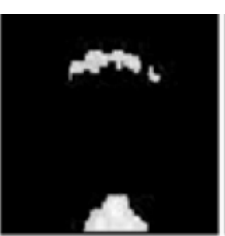

(c) 74

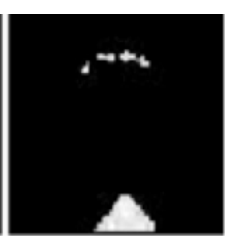

(d) 79
FIGURA 2. Comportamiento de un caso no ideal.

Se puede observar cómo la sustitución por cero de los pixeles no es simétrica. La Figura 2.c y 2.d muestran el caso cuando el valor del umbral fue 74 y 79, respectivamente. Finalmente, los pixeles de la parte superior del cráneo se sustituyeron por cero cuando el valor umbral fue 84, el cual fue asignado a ATO. En este caso, la diferencia en tonos de gris entre mi y ATO fue de 63, el cual es mayor con respecto al del caso anterior.

TABLA 3. Comparación de valores mi, MI y ATO de un caso ideal y no ideal

\begin{tabular}{|c|c|c|c|}
\hline Figura & mi & MI & ATO \\
\hline 1 & 13 & 40 & 40 \\
\hline 2 & 21 & 65 & 84 \\
\hline
\end{tabular}

La Tabla 3 muestra los valores mi, MI y ATO de las Figuras 1 y 2. Como puede observarse, los valores del caso ideal (Figura 1) son menores con respecto a los del caso no ideal (Figura 2).

La Figura 3 muestra los histogramas de los valores de los cráneos ideales, no ideales e infiltrados. En general, un corrimiento a la derecha de los valores mi y MI de los cráneos no ideales e infiltrados con respecto a los ideales es observado. Entre los histogramas de los cráneos ideales y no ideales hay un leve traslape entre los intervalos que determinan si el cráneo tiene o no una enfermedad ósea. Sin embargo, el corrimiento para los cráneos infiltrados es más notorio ya que los mi están en el rango 34-42 y los MI en el rango 98-132, los cuales no se traslapan con ningún rango de los cráneos ideales y no ideales. 
Esta es la razón por la cual decidimos usar los ITGs para diferenciar mediante colores los casos libres de metástasis e infiltrados.
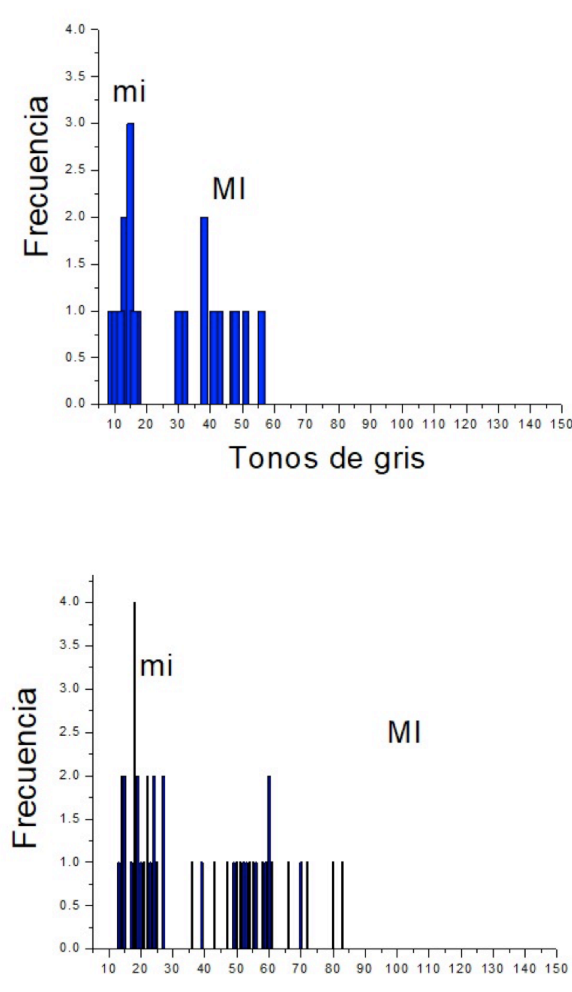

b)

Tonos de gris

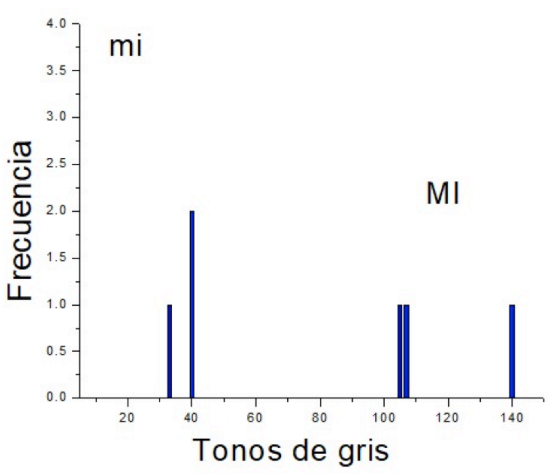

c)

FIGURA 3. Histogramas de los valores mi y MI de, a) cráneos ideales, b) cráneos no ideales y c) cráneos infiltrados.

La Tabla 4 muestra los resultados de los cráneos no ideales para el valor medio y la desviación estándar de mi, MI, y ATO. También muestra los ITGs, los cuales fueron hallados de la misma manera como se obtuvieron los de la Tabla 2. En este caso el intervalo más importante es el ITG 5 ya que con él se determina si el cráneo analizado pertenece o no a esta clasificación. Debido a que los intervalos 5 y 6 se interceptan optamos por reasignarlos a un solo intervalo, el 5'. Los límites de los ITGs 6' y 7' no fueron obtenidos en función del valor medio y la desviación estándar de la muestra sino que fueron propuestos. Igual que antes, las primas son solo para diferenciarlos de los intervalos 5, 6 y 7, y para no perder la continuidad de la numeración. Para este caso particular el intervalo 87-255 (rango de tonos de gris no observado en los cráneos no ideales) se dividió entre dos y el resultado se asignó a cada uno de los dos intervalos. De manera similar que en la Tabla 2, los intervalos 6' y 7' juegan un papel importante ya que en caso de que la imagen muestre alguno de los colores correspondientes a estos dos intervalos, indicarán una posible anomalía ósea en la ROI, la cual se tendrá que analizar más detalladamente para saber de qué tipo es.

TABLA 4. ITGs para los cráneos no ideales (los intervalos 5 y 6 fueron reasignados a un solo intervalo, ITG 5')

\begin{tabular}{|c|c|c|c|}
\hline No. de ITG & mi & MI & ATO \\
\hline Media & $\mathrm{mi}_{\mathrm{s}}=17.27$ & $\mathrm{MI}_{\mathrm{s}}=52.65$ & $\mathrm{ATO}_{\mathrm{s}}=75$ \\
\hline $\begin{array}{c}\text { Desviación } \\
\text { estándar }\end{array}$ & 5.31 & 13.85 & 11.12 \\
\hline ITG 1 & $0-11$ & & \\
\hline ITG 2 & $12-23$ & & \\
\hline ITG 3 & & $24-38$ & \\
\hline ITG 4 & & $39-66$ & \\
\hline ITG 5 & & & $63-67$ \\
\hline ITG 6 & & & $64-86$ \\
\hline ITG 5' & & & $67-86$ \\
\hline ITG 6' & & & $87-171$ \\
\hline ITG 7 & & & $172-255$ \\
\hline
\end{tabular}

Otro caso analizado es cuando el cráneo esta infiltrado. La Tabla 5 muestra los resultados de los cráneos infiltrados y la Tabla 6 muestra los intervalos obtenidos. Estos se muestran con el único propósito de compararlos con aquellos presentados en las Tablas 1 y 4 . Como se puede observar, los valores mi, MI y ATO son mayores comparados con los casos ideal y no ideal. 
TABLA 5. Valores obtenidos de mi, MI y ATO para los cráneos infiltrados

\begin{tabular}{|c|c|c|c|}
\hline $\begin{array}{c}\text { No. de } \\
\text { muestras }\end{array}$ & mi & MI & ATO \\
\hline 1 & 40 & 107 & 280 \\
\hline 2 & 40 & 140 & 237 \\
\hline 3 & 33 & 105 & 253 \\
\hline Media & $\mathrm{im}_{\mathrm{s}}=37.66$ & $\mathrm{IM}_{\mathrm{s}}=117.33$ & $\mathrm{ATO}_{\mathrm{s}}=256$ \\
\hline $\begin{array}{c}\text { Desviación } \\
\text { Estándar }\end{array}$ & 4.04 & 19.65 & 22 \\
\hline
\end{tabular}

TABLA 6. ITGs de los cráneos infiltrados (el ITG 5 fue dividido en dos, ITG 5 ' e ITG 6', con el fin de tener siete intervalos)

\begin{tabular}{|c|c|c|c|}
\hline No. de ITG & mi & MI & ATO \\
\hline ITG 1 & $0-33$ & & \\
\hline ITG 2 & $34-42$ & & \\
\hline ITG 3 & & $43-97$ & \\
\hline ITG 4 & & $98-136$ & \\
\hline ITG 5 & & & $137-233$ \\
\hline ITG 5' & & & $137-184$ \\
\hline ITG 6' & & & $185-233$ \\
\hline ITG 7' & & & $234-292$ \\
\hline
\end{tabular}

Los resultados mostrados en las tablas anteriores indican que los valores mi, MI y ATO, y por lo tanto los ITGs, son función de la "salud" del cráneo ya que según se alejan del caso ideal, sus tonos de gris se incrementan.

Una región con un comportamiento similar al de los cráneos fue la pelvis. Esta es una región con una alta probabilidad de padecer enfermedades de tipo degenerativas como osteopenia u osteoporosis. Estas enfermedades ocasionaron en algunos casos que se observaran amplias regiones con tonos de gris que no correspondían ni a metástasis óseas pero tampoco se ajustaban a la clasificación ideal, de ahí que se clasificaron como no ideales. Nuevamente, de la misma manera que los cráneos, ambas clasificaciones están libres de metástasis.
TABLA 7. Intervalos para la región de la pelvis.

\begin{tabular}{|c|c|c|}
\hline Intervalos & Caso ideales & Caso no ideales \\
\hline ITG 1 & $0-11$ & $0-11$ \\
\hline ITG 2 & $12-20$ & $12-18$ \\
\hline ITG 3 & $21-48$ & $19-41$ \\
\hline ITG 4 & $49-74^{*}$ & $42-63$ \\
\hline ITG 5 & $75-125$ & $64-85^{*}$ \\
\hline ITG 6 & $126-200$ & $86-150$ \\
\hline ITG 7 & $201-255$ & $151-255$ \\
\hline
\end{tabular}

De las 43 pelvis analizadas 15 fueron clasificadas como ideales, 21 como no ideales y 5 como infiltradas. Los ITGs hallados para los dos casos, ideales y no ideales, se muestran en la Tabla 7, donde se observa que en las columnas 2 y 3 los tonos de gris mayores al límite superior del intervalo ITG 4 y 5, respectivamente, indicarán alguna anomalía ósea en progreso. Es decir, cualquier pixel con tonos de gris mayores a 74 y 85 (solo para resaltarlos aparecen con un asterisco en la tabla), probablemente indicarán el inicio de una metástasis ósea o alguna otra enfermedad ósea en progreso. No se presentan resultados para las pelvis infiltradas ya que se aplicará automáticamente el criterio mencionado en el párrafo anterior.

Con una propuesta similar a la de los cráneos y la pelvis, los valores de los ITGs para los hombros y el tórax fueron obtenidos. Estos se muestran en las Tablas $8 \mathrm{y}$ 9, respectivamente. Al igual que en la tabla anterior, los asteriscos solo son para resaltar los valores a partir de los cuales es probable que se encuentre alguna anomalía ósea.

En los casos del tórax, la pelvis y los hombros, además de usar la técnica de sustituir por ceros el valor de los pixeles se usó también la técnica de comparación para obtener los valores mi y MI. La segunda técnica se usó porque existe simetría axial en estas regiones, así que los tonos de gris del lado derecho se compararon con los tonos de gris del lado izquierdo para hallar los valores mi y MI. 
TABLA 8. Intervalos para la región de los hombros

\begin{tabular}{|c|c|c|}
\hline Intervalos & Caso sano & Caso infiltrado \\
\hline ITG 1 & $0-33$ & $0-46$ \\
\hline ITG 2 & $34-46$ & $47-78^{*}$ \\
\hline ITG 3 & $47-65$ & $79-113$ \\
\hline ITG 4 & $66-78^{*}$ & $114-148$ \\
\hline ITG 5 & $79-100$ & $149-183$ \\
\hline ITG 6 & $101-150$ & $184-218$ \\
\hline ITG 7 & $151-255$ & $219-255$ \\
\hline
\end{tabular}

TABLA 9. Intervalos para la región del tórax

\begin{tabular}{|c|c|c|}
\hline Intervalos & Caso sano & Caso infiltrado \\
\hline ITG 1 & $0-21$ & $0-29$ \\
\hline ITG 2 & $22-29$ & $30-52^{*}$ \\
\hline ITG 3 & $30-35$ & $53-93$ \\
\hline ITG 4 & $36-48$ & $94-134$ \\
\hline ITG 5 & $49-52^{*}$ & $135-175$ \\
\hline ITG 6 & $53-100$ & $176-216$ \\
\hline ITG 7 & $101-255$ & $217-255$ \\
\hline
\end{tabular}

\section{RESULTADOS}

El análisis de una ROI usando el método de sustitución de los tonos de gris de los pixeles es una tarea tediosa que consume tiempo, por lo que no sería recomendable su uso en centros médicos con alto volumen de pacientes o con recursos limitados. Para agilizar el proceso, coloreamos la imagen de acuerdo a los ITGs obtenidos para cada región. La Figura 4 muestra la interfaz empleada para asignar colores. Los intervalos mostrados del lado derecho de la interfaz ya incluyen los valores obtenidos para los cráneos infiltrados.

El caso mostrado en la Figura 4 describe perfectamente el objetivo principal del software empleando la técnica comentada y que es la de mostrar que puede funcionar para determinar la metástasis ósea temprana. La imagen en tonos de gris de la izquierda, muestra claramente una ligera acumulación de marcador que indica una metástasis en progreso. Sin embargo, a partir de esa imagen sería aventurado asegurar que del lado derecho también hay una metástasis en progreso.
Al observar la imagen en color notamos en la imagen de la derecha dos regiones amarillas, tanto del lado derecho como del izquierdo. Esta semejanza en color podría ayudar a tomar una decisión más acertada. En este caso, la igualdad en color nos indica que del lado derecho hay una región con alta probabilidad de padecer una metástasis, quizás lo que se observa es el inicio de una metástasis, es decir, una metástasis temprana.

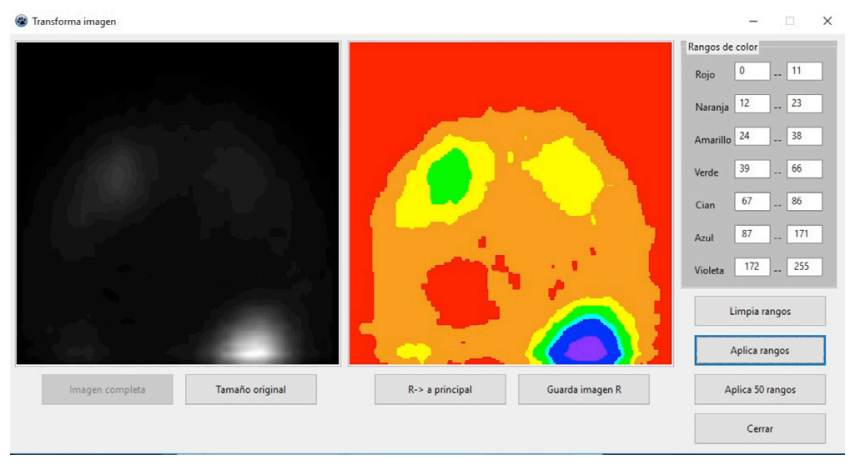

FIGURA 4. Interfaz empleada para asignar colores en función de los ITGs para el caso de cráneos no ideales.

TABLA 10. Propuesta para asignación de colores en un cráneo ideal.

\begin{tabular}{|c|c|c|}
\hline Color & $\begin{array}{c}\text { Tono de } \\
\text { gris inicial }\end{array}$ & $\begin{array}{c}\text { Tono de } \\
\text { gris final }\end{array}$ \\
\hline Rojo & 0 & $\begin{array}{c}\text { Límite superior } \\
\text { ITG1 }\end{array}$ \\
\hline Naranja & $\begin{array}{c}\text { Límite inferior } \\
\text { ITG2 }\end{array}$ & $\begin{array}{c}\text { Límite superior } \\
\text { ITG2 }\end{array}$ \\
\hline Amarillo & $\begin{array}{c}\text { Límite inferior } \\
\text { ITG3 }\end{array}$ & $\begin{array}{c}\text { Límite superior } \\
\text { ITG3 }\end{array}$ \\
\hline Verde & $\begin{array}{c}\text { Límite inferior } \\
\text { ITG4' }\end{array}$ & $\begin{array}{c}\text { Límite superior } \\
\text { ITG4, }\end{array}$ \\
\hline Cian & $\begin{array}{c}\text { Límite inferior } \\
\text { ITG5, }\end{array}$ & $\begin{array}{c}\text { Límite superior } \\
\text { ITG5, }\end{array}$ \\
\hline Azul & $\begin{array}{c}\text { Límite inferior } \\
\text { ITG6' }\end{array}$ & $\begin{array}{c}\text { Límite superior } \\
\text { ITG6 }\end{array}$ \\
\hline Violeta & $\begin{array}{c}\text { Límite inferior } \\
\text { ITG7 }\end{array}$ & $\begin{array}{c}\text { Límite superior } \\
\text { ITG7 }\end{array}$ \\
\hline
\end{tabular}

La Tabla 10 muestra el criterio usado para asignar los colores a cada intervalo de nivel de gris para el caso de cráneos ideales. Como se puede observar, para realizar la asignación de colores se consideraron los límites inferior y superior de los ITGs hallados, y por consi- 
guiente el promedio y la desviación estándar de los tonos de gris de las ROIs. La Tabla 11 muestra la asignación de colores usando la propuesta mostrada en la Tabla 10. La Figura 5 muestra imágenes en color de los cráneos ideal, no ideal e infiltrado, como resultado de aplicar los ITGs obtenidos en las Tablas 2, 4 y 6.

TABLA 11. Resultado de aplicar la propuesta mostrada en la Tabla 10 (los valores fueron tomados de la Tabla 2)

\begin{tabular}{|c|c|c|}
\hline Color & $\begin{array}{c}\text { Nivel de } \\
\text { gris inicial }\end{array}$ & $\begin{array}{c}\text { Nivel de } \\
\text { gris final }\end{array}$ \\
\hline Rojo & 0 & 10 \\
\hline Naranja & 11 & 16 \\
\hline Amarillo & 17 & 32 \\
\hline Verde & 33 & 57 \\
\hline Cian & 58 & 124 \\
\hline Azul & 125 & 191 \\
\hline Violeta & 192 & 255 \\
\hline
\end{tabular}

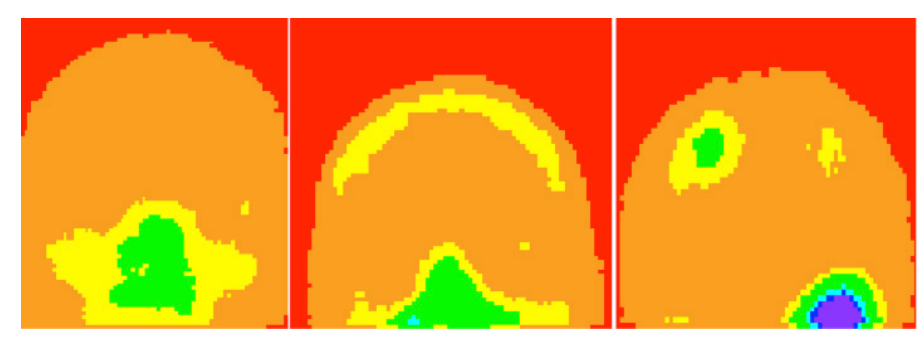

FIGURA 5. Se muestran imágenes en colores para un cráneo ideal, no ideal e infiltrado.

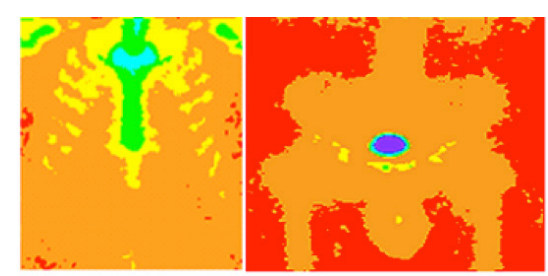

(a)

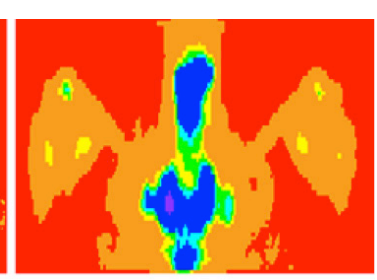

(c)
FIGURA 6. Imágenes en color de a) tórax, b) pelvis y c) hombros, libres de metástasis.

Una propuesta similar a la de los cráneos y la pelvis se empleó para el tórax y los hombros. La Figura 6 muestra tres ROIs libres de metástasis óseas y la Figura 7 muestra tres ROIs infiltradas. La Figura 7.a muestra una metástasis ósea en un arco costal en color verde, en la Figura 7.b las metástasis están en cian y en la Figura 7.c las metástasis están en color azul. Los diferentes colores para las metástasis se deben a que las ITG's tienen diferentes ROI's.

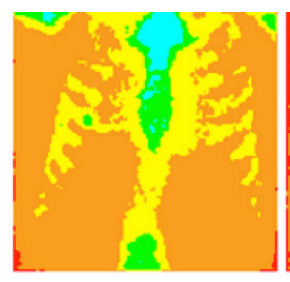

(a)

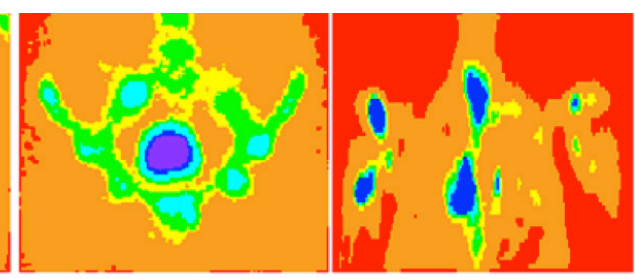

(b) (c)
FIGURA 7. Imágenes en color de a) tórax, b) pelvis y c) hombros, infiltradas.

\section{DISCUSIÓN}

La imagen de un gammagrama óseo suele ser almacenada en un archivo donde un pixel puede guardar cuando menos 1024 valores ( $2^{10}$ números, bit depth). Para un cráneo sano del tipo ideal se encontró que los tonos de gris de su gammagrama óseo no son mayores a 60 tonos. Para el caso de un cráneo sano del tipo no ideal los tonos de gris se incrementaron, pero no son mayores a 100. Solo hay dos regiones donde el tono de gris puede tomar el valor más alto (1024), siendo éstas la región donde se inyecta el marcador y la vejiga, que es el lugar donde se almacena el marcador no absorbido. Sin embargo estas dos regiones no son de interés para el diagnóstico ya que siempre tendrán los tonos de gris más altos y nunca indicarán una metástasis.

Un objetivo del software que se está desarrollando, es determinar la metástasis ósea temprana y otras enfermedades degenerativas, como osteopenia u osteoporosis. Para dichas enfermedades, tampoco se halló que los tonos de gris alcanzaran el valor de 100. Debido a que los tonos de gris de un gammagrama son bajos, aun cuando la profundidad del bit es alta, no se explota al máximo esta cualidad. Si a esto le agregamos el problema de que el ojo humano solo puede resolver en el mejor de los casos 32 tonos de gris $\left(2^{5}\right)$ de $256^{[8]}$, será 
imposible que observando la imagen en tonos de gris se observen las anomalías óseas deseadas. Es por eso que decidimos usar colores para resolver el problema.

Algunos equipos de visualización despliegan gammagramas en formato DICOM automáticamente de 0 a 255 tonos sin considerar los bits del formato original. Tal característica puede ser una desventaja cuando el rango de valores de gris de la imagen es bajo, como ocurre con los gammagramas, primero porque se ven pixelados y segundo porque el operario podría confundirse y diagnosticar erróneamente una zona con valores bajos al visualizarse en 256 tonos, el conocimiento de los ITGs evita este problema. Existen diferencias en los ITGs entre tipos de gammagramas, por ejemplo, los valores para el tipo Bone Amed BC Off son ligeramente mayores que los del $3 \mathrm{Hr}$ Whole-Body. Hasta ahora, no se ha validado la clasificación de ITGs en otras cámaras gamma, por lo que será necesario también calibrar cada cámara. Así que será necesario agregar otras calibraciones al software si se desea analizar otros tipos.

Aunque los ITG fueron calculados empleando gammagramas libres de metástasis, es necesario usar muestras totalmente libres de cualquier otra enfermedad ósea para obtener ITG lo más preciso posible, tal como ocurrió con los cráneos ideales. Las Figuras 1 y 2 fueron obtenidas de cráneos diagnosticados como libres de metástasis óseas por lo que creemos que lo observado en la Figura 2 y los 29 casos restantes corresponden a enfermedades diferentes, aunque no sabemos cuáles son debido a que no esperábamos estos resultados; por lo tanto, no se tuvo cuidado de hacer exámenes adicionales a los pacientes. Hasta ahora nos hemos enfocado a la determinación de los intervalos de tonos de gris que diferencian huesos sanos de los no sanos y como hemos visto los resultados son alentadores.

Con la finalidad de determinar la metástasis ósea temprana u otras enfermedades, es necesario incrementar sustancialmente la muestra de gammagramas óseos analizados para hallar en particular el mejor valor de MI, ya que valores mayores a él indicarán los inicios de la metástasis temprana o de otra enfermedad ósea.

A diferencia de otros softwares que analizan el gammagrama completo, por ejemplo, Bonenavi [2, 9, 10] y Exini [3, 11], el que está en desarrollo parecería que tiene una desventaja al tener que realizar el análisis por regiones y tener que hallar los ITGs. Sin embargo, después de realizar la calibración y que el análisis se haga automáticamente la desventaja desaparecerá.

\section{Limitaciones del estudio}

Idealmente, el número de gammagramas que se deben usar para este tipo de estudios debe ser del orden de cientos de casos, incluyendo casos representativos hallados en rutinas clínicas, además de contar con un exacto e independiente método como estándar de oro. Una verificación histológica de cada punto caliente hallado en los gammagramas óseos podría haber sido un mejor estándar de oro que el análisis visual que realizaron los tres especialistas de medicina nuclear.

\section{CONCLUSIONES}

Un método cuantitativo para detectar metástasis esquelética en gammagramas óseos fue presentado. Para el análisis, los gammagramas fueron segmentados en ROIs donde intervalos de tonos de gris fueron hallados estadísticamente. Después, se asignó falso color a la imagen de acuerdo a los ITGs establecidos con ayuda del valor medio y la desviación estándar de los niveles de gris de las ROIs de la muestra. El método funcionó bien, ya que existe un intervalo considerable entre los tonos de gris de los casos sanos e infiltrados; sin embargo, como el objetivo del software es también determinar la metástasis ósea temprana y otras enfermedades óseas, es necesario aumentar el tamaño de la muestra y asegurarse de que los gammagramas analizados estén libres de cualquier enfermedad ósea con el fin de determinar el mejor valor de los ITGs, ya que un valor erróneo podría conducir a un mal diagnóstico. 
Antes de probar el software en un centro oncológico es necesario asegurarse de que los intervalos de tonos de gris hallados en este trabajo aplican para cualquier tipo de cámara gamma. También es necesario determinar los intervalos de tonos de gris para otro tipo de gammagrama, por ejemplo, el Bone Amed BC off. Hasta el momento, los resultados son alentadores por lo que la técnica podría ser usada en el futuro para asistir a los expertos en el diagnóstico de las metástasis óseas y algunas enfermedades degenerativas.

\section{RECONOCIMIENTOS}

Los autores agradecen al Centro Médico Nacional "La Raza" del Instituto Mexicano del Seguro Social de la Ciudad de México y a la Dra. Blanca Zapote

Hernández las facilidades otorgadas para la obtención de los gammagramas óseos.

Los autores agradecen también a los árbitros por sus útiles y atinados comentarios y sugerencias para mejorar el trabajo. 


\section{REFERENCIAS}

[1] Zafeirakis A, Scoring systems of quantitative bone scanning in prostate cancer: historical overview, current status and future perspectives, Hell J Nucl Med, 2014; 17(2), 136-144. doi: 10.1967/ $\underline{\mathbf{0 0 0 2 4 4 9 9 1 0 1 3 4}}$

[2] Imbriaco M, Larson S, Yeung H, Mawlawi O, Erdi Y, Venkatraman E, Scher $\mathrm{H}$, A new parameter for measuring metastasic bone involvement by prostate cancer: the bone scan index. Clin Cancer Research 1998; 4: 1765-1772.

[3] Sadik M, Hamadeh I, Nordblom P, Suurkula M, Hoglund P, Ohlsson M, Edenbrandt L, Computer-assisted interpretation of planar whole-body bone scans, J Nucl Med 2008; 49: 1958-1965. doi: 10.2967/jnumed.108.055061.

[4] Jaramillo-Núñez A, Zapote-Hernández B, Sánchez- Rinza B, TitlaTlatelpa JJ. Determinación de los rangos ideales de tonos de gris para una muestra de 59 gammagramas óseos. Anales de Radiología México 2016;15(4):345-356.

[5] Zapote-Hernández B, Cruz-Santiago JC, González-Vargas E, Jaramillo-Núñez A., Concordancia diagnóstica entre los métodos visual e informático en la detección de metástasis por gammagrafía ósea en cáncer de próstata. Anales de Radiología México 2016;15(2):111-119.

[6] Alberto Jaramillo Núñez and J. Carlos Gómez-Conde, Método para incrementar la sensibilidad diagnóstica del gammagrama óseo, Anales de Radiología México, 2015; 14: 11-19.
[7] Zapote-Hernández B., Diagnostic concordance between the visual analysis and by software in bone metastases detection by bone scintigraphy in prostate cancer, tésis de especialidad, UNAM, Fac. de Medicina, 2016.

[8] Bourne R, Fundamentals of digital imaging in medicine, SpringerVerlag, London, 2010.

[9] Sabbatini P, Larson SM, Kremer A, et al. Prognostic significance of extent of disease in bone in patients with androgen-independent prostate cancer. J Clin Oncol. 1999;17: 948-957.

[10] Mitsuru Koizumi, Kei Wagatsuma, Noriaki Miyaji, Taisuke Murata, Kenta Miwa, Tomohiro Takiguchi, Tomoko Makino, Tasamichi Koyama, Evaluation of a computer-assisted diagnosis system, BONENAVI version 2, for bone scintigraphy in cancer patients in a routine clinical setting, Ann Nucl Med (2015), 29; 138-148. doi: 10.1007/s12149-014-0921-y

[11] Sadik M, Suurkula M, Hoglund P, Jarund A, Edenbrandt L. Improved classifications of planar whole-body bone scans using a computer-assisted diagnosis system: a multicenter, multiplereader, multiple-case study. J Nucl Med. 2009;50: 368-375. doi: 10.2967/ jnumed.108.058883 